des Giftes hervor, die binnen 12 Stunden wieder verschwinden oder zum Tode führen. Bei starken Dosen erkranken die Pferde schon nach 10 Minaten und sterben nach einigen Stunden.

Nach Zanon wird das Fagin erhalten, wenn man die Presskuchen mit Wasser auszieht, den emulsionsähnlichen Auszug mit Kalkerdehydrat vermischt zur Trockne bringt und den Rǚckstand mit kaltem Spiritus auszieht. Der Alkohol wird zum grössten Theile wieder abdestillirt, die Flüssigkeit filtrirt und zur Trockne verdunstet. Hieraus zieht Wasser dann das Fagin mit schöner gelber Farbe aus und lässt es als eine schöne gelbe zöhe Masse zurïck, die alkalisch reagirt, 'süsslich und eigenthümlich schmeckt, und mit Schwefelsäure ein in Prismen krystallisirendes graugelbes Salz liefern soll. Zugleich aber giebt er an, dass dieselbe gelbe Basis durch Alkalien ans einer Emulsion der Bucheln gefällt werde, was ihrer Löslichkeit in Wasser zu widersprechen scheint.*)

Hirschberg.

\title{
Die Rinderpest.
}

Keine Krankheit unserer landwirthschaftlichen Hausthiere ist geeigneter das Interesse und die Aufmerksamkeit sowohl des Einzelnen, als auch des ganzen Staates auf sich zu lenken, als die Rinderpest, da sie da, wo sie einmal zum Ausbruch gekommen und einen gewissen Grad ron Ausbreitung gewonnen, schwer zu tilgen und wegen ihrer räuberischen Eingriffe in das Privat - und Staats - Eigenthum als eine wahre Landes-Calamität zu betrachten ist. Man sieht daher auch, wie sorgsam die Regierungen derjenigen Länder, die der Gegend der herannahenden Rinderpest-Verwüstungen am nëchsten liegen, Anstalten treffen, um den bösen Feind fern

*) Man vergleiche über die Bestandtheile von Fagus sylvatica L. Rochleder's Chemie und Physiologie der Pflanzen 1858. Seite 77. 
zu halten von ihren Landen, und bemüht sind, wenn er in ihnen eingetroffen, als solchen $z \mathfrak{u}$ bekämpfen und $\mathrm{zu}$ vertilgen von dem Boden, den er so eben angegriffen. Den Ort der Entstehung dieser furchtbaren Seuche anlangend, so ist so viel feststehend, dass ihre Heimath die russischen Steppen sind. Von hieraus, wo sie sich spontan entwickelt, kann sie auf dem Wege der Ansteckung in alle Länder der Erde verschleppt werden, und in Europa sind wohl wenige Gegenden anzutreffen, die nicht schon früher die verderblichen Folgen ihres gekosteten Giftes empfunden haben. Um die Art und Weise, wie die Rinderpest in Holland eingeschleppt ist, kennen zu lernen, müssen wir erst erfahren, dass sie kurz zuvor in England ausgebrochen war. Genaue Nachweise, wie und auf welche Weise die Seuche nach England gelangt ist, sind bis jetzt nicht geliefert worden. Constatirt ist nur, dass am 29. Mai 1865 ein Transport von 320 Ochsen, der in Riga eingeschifft war, in Zeit von 6 Tagen, also innerhalb der für die Rinderpest allgemein geltenden Incubationszeit nach Hull gelangte. Von diesen 320 Ochsen wurden in Hull und dessen Umgegend 145 verkauft, die übrigen 175 aber nach London versandt. Als die Zeit, in der das erste Auftreten der Rinderpest bemerkt worden, ist der 27. Juni 1865 bezeichnet; an diesem Tage wurden 6 auf dem Londoner Viehmarkte gekaufte Kühe in Mr. Nicholl's Viehhaltung Nr. 15. Park Place, Liverpool Read, Islington von der Krankheit ergriffen. Obgleich diese 6 Kühe in einem Quarantaine - Stall standen, ging die Krankheit dennoch auf andere Kühe über; 115 sind gestorben. Unter denjenigen Ländern, die die ungeheure Hauptstadt Englands mit Schlachtvieh versorgen, steht Holland verhältnissmässig obenan. Während Hamburg, das an dieser Versorgung Londons mit Schlachtvieh einen nicht unerheblichen Antheil nimmt, in den ersten 6 Monaten 18653313 Stück Rindvieh nach dieser Stadt ausführte, hat Rotterdam allein 18951 Stück dorthin versandt. Durch den regen Viehverkehr, der zwischen Holland und England besteht, so wie dadurch, dass die holländischen Häfen sich in einer nur geringen Entfernung von den englischen befinden, können die 
nicht abgesetzten Rinder sehr leicht zurückgenommen werden. Diese Verhältnisse, durch welche, wie leicht einzusehen, günstige Bedingungen für die Verschleppung der Rinderpest von England nach Holland geschaffen werden, haben die erste Veranlassung zu der gegenwärtig noch in Holland herrschenden Seuche gegeben. Ueber die Art, wie die Rinderpest in Holland eingeschleppt worden, sind folgende Nachrichten bekannt. Im Juni wurden dem Mr. Defries jun. in London von seinem Vater aus Holland 23 fette Ochsen mit dem Auftrage zugesandt, dieselben in England zu verkaufen. Die Ochsen wurden zu wiederholten Malen zum Verkauf' gestellt, weil der für dieselben gebotene Preis von $131 / 2$ Pf. St. pro Stück dem Eigenthümer nicht convenirte. 13 Ochsen wurden am 22., 26. und 29. Juni zu Markt gebracht, die übrigen 10 einen Markttag um den andern. Während dieser Zeit standen die Ochsen in Mrs. Nicholl's Schuppen oder weideten auf den Feldern derselben, unter deren Vieh die Rinderpest, obgleich man sie noch nicht erkannt hatte, ausgebrochen war. Sowohl bei Mrs. $\mathrm{Nicholl}$, als auf dem Markte konnte es nicht fehlen, dass die Ochsen mit vielem Vieh, sowohl einheimischen, als auch eingeführten, in Berührung kamen. Herr Defries sen., der die geforderten Preise nicht erhalten konnte, liess die Ochsen nach Holland zurückbringen, und fand deren Verschiffung am 2. Juli statt. Herr Simonds, der das Amt eines Inspectors bekleidete, bemerkte wohl, dass die Ochsen nicht gesund waren, hatte jedoch nicht die Macht, sie zurückzuhalten. Sehr bald, nachdem die Ochsen in Holland angekommen waren, erkrankten sie auffällig und schon in den nächsten Tagen crepirten 21 derselben. Da man bei der Beurtheilung dieser Krankheit keinen Argwohn hegte, so wurden alle Vorsichtsmassregeln vernachlässigt; auch unterliess man es, Anzeige davon bei den Behörden zu machen. Die ersten Opfer der Ansteckung durch die Ochsen des Herrn Defries waren Rindviehstücke des Herrn Vanderwalden. Die kranken Ochsen des Herrn D. wurden nämlich auf eine Weide getrieben, auf der sie mit Rindvieh des Herrn V. zusammenkamen. Letzterer Besitzer verkaufte, ehe die 
Thatsachen allgemein bekannt wurden, 20 von den inficirten Rindern und verschleppte auf diese Weise die Seuche nach verschiedenen Richtungen. Feststehende Thatsache ist also, dass durch Herm Defries sen. die Rinderpest von England nach Holland verschleppt worden. Durch die That, dass Herr Defries seine in London nicht abgesetzten Ochsen nach Holland zurückbringen liess und dadurch die Sevche dorthin verschleppte, hat er sich verewigt. Seinen Namen wird die Zukunft zu nennen wissen, so lange die Blätter da, auf denen die Geschichte der Rinderpest geschrieben steht. Wäre dem Herrn Defries das Ereigniss, dass er mit der Zuriickbringung seiner Ochsen nach Holland zugleich die Rinderpest mit dorthin versehleppen würde, bekannt gewesen, sein Name würde fluchwürdiger, wie der des Herostrat sein, der einst von eitler Ehrsucht getrieben, die Brandfackel ergriff, mit der er des Alterthums stolzesten Bau in Flammen setzte. Nachdem, wie vorhin bemerkt, die 20 inficirten Rinder des Herrn Vanderwalden verkauft und so die Seuche nach verschiedenen Richtungen verschleppt worden, gewann sie schnell an Ausbreitung. Inficirte Thiere, die sich im Incubationsstadium der Krankheit befanden, wurden Woche für Woche nach England ausgeführt und dadurch immer von Neuem Gelegenheit zum Ausbruch der Rinderpest daselbst geboten. Die in Norfolk und Suffolk vorgekommenen Seuchenfälle sind ganz bestimmt durch die Einfuhr von holländischem Vieh verursacht worden. Erst im März 1866 erliess England, nachdem es also 10 Monate lang Rindvieh aus einem verpesteten Lande bezogen, eine Verordnung, in der die Einfuhr von Rindvieh aus Holland verboten wurde. Wenn man bedenkt, dass die beiden Länder Holland und England schon in früheren Jahren arge Verwüstungen durch die Rinderpest erlitten, dann ist es schwer zu begreifen, dass dieselben nicht durch die Erfahrungen der Vorzeit belehrt ein wachsames Auge auf die Ankunft derselben richteten und es an Massregeln fehlen liessen, durch die sie dieselben bekämpften und vertilgten, sobald sie bei thnen eingetroffen. Im Jahre 1745, wo die Rinderpest in Holland über 200 Hëupter forderte, wurde sie 
in demselben Jahre von hier aus nach England verschleppt. Diese Verschleppung der Seuohe geschah angeblich durch 2 weisse Kälber, die ein Farmer in Poplar aus Holland zu Züchtungazwecken ankaufte, nach einer anderen Angabe durch den Verkauf einer Parthie Häute aus der Provinz Seeland, deren Verkauf dort verboten war und die den gesetzlichen Bestimmungen zu Folge hätten vergraben werden sollen.

Die Rinderpest, welche später über das ganze Königreich rerbreitet war, herrschte über 12 Jahre im Lande, und die Anzahl der Opfer, welche sie forderte und die nicht einmal annähernd bestimmt werden konnte, war eine ganz enorme.

In den Jahrén von 1770 bis 1772 waren die Verheerungen der Rinderpest in Holland ungeheuer, bei 300000 Stück fielen ihr als Opfer; bis zum Jahre 1776 setzte sie ihre Verheerungen mit solcher Wuth fort, dass dieser durch Viehzucht sonst so blühende Staat von seinem Viehstande ganz entblösst wurde. Dieses Unglück bewog auch damals die Staatsbehörden daselbst, auf die Auffindung eines specifischen Heilmittels gegen diese Pest einen sehr hohen Preis zu setzen, um den sich jedoch Niemand zu bewerben wagte. Wie sehr man übrigens auch jetzt noch an die Möglichkeit der Auffindung eines Heilmittels oder einer Curmethode der Rinderpest zu. glauben scheint, geht daraus hervor, dass sich aus verschiedenen Ländern eine gewisse Anzahl Aerzte, sowohl allopathischer als homöopathischer, in Holland aufhält, um sich in der Behandlung der Rinderpest zu versuchen; von keinem derselben ist aber bekannt geworden, dass seine Behandlung der Rinderpest zu etwas Erspriesslichem und Heilsamen geführt habe. Als in England der Ausbruch der Rinderpest constatirt war, wurde die Frage: ob man durch schnelles Tödten der Erkrankten eine baldige und gründliche Tilgung der Seuche erzielen, oder ob man durch Behandlung der Erkrankten in grossen Hospitälern eine Heilung derselben erstreben solle, aufgeworfen. Die Professoren der Thierarzneischulen in London und Edinburg, welche mit der Literatur der Rinderpest bekannt sind, sprachen sich ohne Ausnahme für die erste Alternative aus und stützten sich auf die Erfolge, welche 
dieses Verfahren in andern Ländern erzielt hat; sie befanden sich mit ihrer Ansicht jedoch in der Minorität, da der grosse Haufe und mit ihm viele Thierärzte lebhaft die Einrichtung von Pestspitälern und die Behandlung der erkrankten Rinder forderten. Die Regierung schwankte in diesem Streite hin und her, rieth im Allgemeinen von Einrichtung von Spitälern ab, ohne jedoch im Stande zu sein, den Gemeinde-Behörden von London die Anlage eines solchen Spitals zn verbieten. Allerlei Motive treten bei den Anhängern der Behandlung der Rinderpest in den Vordergrund. Religiöse und moralische Bedenken werden hervorgezogen, um den Beweis zn liefern, dass der Mensch die Verpflichtung habe, die erkrankten Thiere durch passende Arzeneimittel zu erhalten und herzustellen, während allgemeines 'Tödten der Erkrankten als eine mit unserer Civilisation unvereinbare Grausamkeit gebrandmarkt wird. Die Selbstüberschätzung einiger englischer Thierärzte, denen in den meisten Fällen die Rinderpest eine terra incognita ist, nimmt hänfig genug den Mund voll, blickt mit einiger Verachtung auf die Thierärzte des Continents und hofft durch allerlei Geheimmittel die glücklichen Erfolge bei der Behandlung $\mathbf{z u}$ erreichen, welche unsere Unwissenheit zu erzielen nicht verstanden hat. Der grosse Haufen der Viehbesitzer endlich sieht nur den allernächsten Vortheil und Nachtheil; was kümmert den Einzelnen, dass eine kranke Kuh den Rindviehbestand seiner ganzen Nachbarschaft in Gefahr bringt. Selbst wenn eine nur geringe Anzahl durch Naturheilung oder durch Arzeneimittel am Leben erhalten wird, hat der betreffende Besitzer Vortheil; ein Tödten der Erkrankten bringt aber gewissen Verlust, da Entschädigangen für die aus sanitätspolizeilichen Gründen getödteten Thiere nicht bezahlt werden. Mit Recht macht Professor Simonds in einem Berichte an den Minister daranf aufmerksam, dass erst die Bezahlung der getödteten Thiere die getroffenen Tilgungsmassregeln zur vollen Wirksamkeit bringen könne. Die von der holländischen Regierung erlassenen Verordnungen in Betreff der Rinderpesttilgung haben sich nichts weniger als erfolgreich bewiesen; denn nicht allein ist keine Verminde- 
rung der Seuche in den inficirten Provinzen erfolgt, sondern auch ein Ausbruch und Weiterverbreiten derselben in seuchefreien Provinzen hat stattgefunden. Wenn auch zeitweilig eine Einschlummerung der Seuche eintritt, so kann man sich doch der Annahme nicht entschlagen, dass sie später wieder erwachen und ihre Verheerungen von Neuem beginnen wird. Einer Commission, welche grösstentheils ans Verwaltungsbeamten, Mitgliedern der Provinzialstände und nur einem Thierarzte bestand, wurde anheimgegeben, sich mit der Auffindung der Mittel und Wege, die zur Tilgung der Rinderpest führen, zu beschäftigen. Es kann nicht in Abrede gestellt werden, dass diese Commission im Allgemeinen das Richtige in Betreff der Lösung ihrer Aufgabe getroffen und auch nicht ermangelt hat, in dem von ihr geforderten Berichte an den König der Niederlande ihren Ansichten Ausdruck zu verleihen. Das Princip, was man in Holland im Auge gehabt, kann nicht für unzweckmässig gehalten werden, nur sind die vielfachen Mängel und Gebrechen abzustellen, an denen die Durchführung der zur Abwehr der Seuche erlassenen Bestimmungen leiden. Die Regierung scheint bisher Bedenken getragen zu haben, in die freiheitlichen Rechte der Gemeinden, in denen das selfgouvernement besteht, mit der gehörigen Energie einzugreifen; wenigstens ist bisher noch keine Ministerialverfügung erlassen worden, die die Provinzialregierungen zur Ergreifung strengerer Massregeln ermächtigt.

Es liegt anf der Hand, dass unter solchen Verhältnissen die Gefahr der Einschleppung in die an Holland grenzenden Kreise in dem Maasse wuchs, in welchem die Verbreitung der Rinderpest in Holland zunahm und sich den preussischen Grenzen näherte. Die lokalen Verhältnisse der Grenzkreise trugen wesentlich noch zur weiteren Steigerung dieser Gefahr bei. Für vorzüglich misslich muss angesehen werden, dass die westliche Grenze gegen Holland sehr schwer zu überwachen ist; eine deutliche Landesgrenze ist wenigstens im Regierungsbezirk Düsseldorf gar nicht vorhanden, holländische und preussische Gehöfte liegen an vielen Stellen dicht neben einander, preussische Bauern besitzen Ackerstiicke in Holland 
und umgekehrt, nicht selten geht die Grenze quer durch ein Gehöft oder durch ein Wohnhaus, Der sehr rege Verkehr zwischen dem preussischen und holländischen Gebiete ist nicht auf bestimmte Zollstrassen beschränkt, sondern durch Nichts gehemmt. Unter diesen Umständen ist es um so mehr anzuerkennen, dass die energischen Massregeln der Regierung den Erfolg hatten, die nicht zu verhütende Einschleppung der Rinderpest auf wenige Seuchenbeerde zu beschränken. In der Zeit von circa 2 Monaten ist die Rinderpest nur an 6 Orten des Regierungsbezirks Düsseldorf und in einer Ortschaft des Regierungsbezirks Münster zum Ausbruch gekommen. In keinem Falle hat sich die Rinderpest nach ihrer amtlichen Feststellung von dem inficirten Gehöfte aus weiter verbreitet; das ist der schlagendste Beweis von der bewährten Zweckmässigkeit und Energie des preussischen Tilgungsverfahrens. Vom 14. December 1866 bis 22. Februar 1867 ist die Rinderpest nur auf 7 Gehöften zum Ausbruch gekommen und der Totalverlust, welcher durch sie herbeigeführt, ist mit 133 Stück Hornvieh angeführt; von diesen 133 sind 3 gestorben und die übrigen 130 , unter denen $18 \mathrm{Er}$ krankte und 112 Gesunde, sind getödtet worden. Die Entschädigungssumme für sämmtliches getödtete Vieh hat 6977 Thlr. betragen. In allen Fällen sind die Sperr - und Desinfections-Masssregeln in der gtrengsten Art und Weise nach dem von dèn Regierungen zu Düsseldorf und Münster vorgeschriebenen Verfahren ausgeführt worden. Ausserdem sind die Grenzen gegen Holland bis auf die Eisenbahnen und grossen Zollstrassen gesperrt, die militairische Grenzbesetzung wurde verstärkt, die eingehenden Guiter werden strenge revidirt, jedes mit Stroh verpackte Colli wird streng zurückgewiesen, die aus Holland eintretenden Personen mussten sich einer Desinfection unterwerfen und die Regierung hatte Thierärzte engagirt, welche in die Postorte stationirt wurden. Hiezu kommt, dass unter der Bevölkerung die grösste Besorgniss und die volle Bereitwilligkeit herrscht, alle Verkehrsbeschränkangen, die zur Abhaltung der Seuche unvermeidlich sind, zu ertragen. Man kann dreist behaupten, dass 
von der ländlichen Bevölkerung wissentlich Nichts geschehn wird, was zur Einschleppung beitragen könnte. So ist es denn glücklich gelungen, die Rinderpestausbrüche in den Grenzkreisen auf wenige Fälle zu beschränken. Wir haben alle Ursache, uns über die Erfolge zu freuen und müssen die Hoffnung aussprechen, dass die strenge Grenzsperre auf das niederländische Gouvernement eine Pression ausübt, welche dasselbe veranlasst, auch in dem eigenen Lande energischere Maassregeln als bisher gegen die Seuche zu ergreifen. Die Symptome der Rinderpest sind folgrende: zuerst Husten, die Milch nimmt ab, später Appetitlosigkeit, 6 Tage lang haben die Thiere einen heiseren Husten, es tritt Abmagerung ein und in 8 Tagen crepiren sie. Die Krankheit hat mit der Maul - und Klauenseuche Aehnlichkeit, im Entstehen ist sie mit Katarrhalfieber am leichtesten zu verwechseln.

Apotheker Schultze in York.

\section{Die Trunksucht und der Săuferwahnsinn in Russland.}

In Folge der grenzenlosen Trunksucht in Russland ist der Säuferwahnsinn bei den Russen im steten Zunehmen begriffen. Während 1862 in 4 Petersburger Civilspitälern 266 Individuen an dieser scheusslichen Krankheit behandelt worden sind, kamen 1863 schon 715 und 1864 sogar 853 Fälle von Delirium vor. Von der Cnmasse, welche in Russland insgesammt von diesem Leiden heimgesucht werden, kommen 35 Proc. auf niedere Beamte, 32 Proc. auf Städter und Handwerker, 13 Proc. auf Tagelöhner u. s. w.

1859 kamen von dem in St. Petersburg consummirten Branntwein 1,68 Eimer auf jeden Einwohner dieser Stadt ohne Unterschied der Person und des Geschlechts.

1866 ergab die Branntweinsteuer in Russland 116 Millionen Rubel Silber. (Petersburger medicinische Zeitung 1867.). Dr. H. Reich. 